Images du travail, travail des images

1 | 2016

Quand les groupes professionnels se mettent en images

\title{
Grand entretien avec Pierre Carles
}

Jean-Paul Géhin, Maxence Lamoureux et Pierre Carles

\section{CpenEdition}

Journals

Édition électronique

URL : http://journals.openedition.org/itti/1349

DOI : 10.4000/itti. 1349

Éditeur

Université de Poitiers

Référence électronique

Jean-Paul Géhin, Maxence Lamoureux et Pierre Carles, "Grand entretien avec Pierre Carles », Images

du travail, travail des images [En ligne], 1 | 2016, mis en ligne le 01 février 2016, consulté le 14 avril

2021. URL : http://journals.openedition.org/itti/1349 ; DOI : https://doi.org/10.4000/itti.1349

Ce document a été généré automatiquement le 14 avril 2021.

Images du travail, travail des images 


\title{
Grand entretien avec Pierre Carles
}

\author{
Jean-Paul Géhin, Maxence Lamoureux et Pierre Carles
}

Pierre Carles est né le 02 avril 1962 ; après des études de journalisme à l'IUT de Bordeaux, il a travaillé dans de nombreuses chaines de télévision. Réalisant une critique des médias de l'intérieur, nombre de ses reportages puis de ces documentaires ont été déprogrammés ou ont connu des difficultés pour être vus à la télévision. De 1993 à 1998, il a réalisé une dizaine de courts métrages pour l'émission Strip tease puis, à partir de 1995, de nombreux documentaires de création produits par une petite société de production indépendante (C-P production) et diffusés en salle de cinéma.

D'abord connu pour sa critique des médias dominants (Pas vu pas pris; Enfin pris?; Fin de concession) il a aussi développé une critique du salariat (avec Christophe Coello et Stéphane Goxe), s'est intéressé aux modes de vie alternatifs (avec Coello/ Goxe et Philippe Lespinasse), à la sociologie (film sur Pierre Bourdieu) et à la politique (documentaires sur les élections). Il a ainsi développé une œuvre cinématographique singulière marquée par sa thématique, la relation entre les mass médias et la politique, mais aussi par sa forme : modalités d'entretien, réflexivité...

\section{Filmographie}

1995 : Juppé, forcément, 31 minutes

1998 : Pas vu pas pris, 90 minutes

2001 : La sociologie est un sport de combat, 146 minutes

2002 : Enfin pris ?, 93 minutes

2003 : Attention danger travail, 109 minutes, réalisé avec Christophe Coello et Stéphane Goxe

2006 : Ni vieux, ni traîtres, 93 minutes, réalisé avec Georges Minangoy, consacré à Action directe

2007 : Volem rien foutre al païs, 107 minutes, réalisé avec Christophe Coello et Stéphane Goxe 
2008 : Qui dit mieux ?, 90 minutes, réalisé avec Christophe Coello et Stéphane Goxe 2008 : Val est vénere, réalisé avec Éric Martin

2009 : Choron, dernière : Vie et mort du professeur Choron et de Charlie Hebdo, réalisé avec Éric Martin, 104 minutes

2010 : Fin de concession, 128 minutes

2012 : Hollande, DSK, etc réalisé avec Julien Brygo et Aurore Van Opstal, 83 minutes

2012 : Tant pis / tant mieux, réalisé avec Philippe Lespinasse et composé de deux épisodes : Bages-Sigean à la voile (2007, 52 minutes) et Gruissan à la voile (2009, 52 minutes)

2014 : Opération Correa - Première partie : les ânes ont soif, 54 minutes. Interviews : Aurore Van Opstal, Nina Faure, Brice Gravelle, Julien Brygo

Ce média ne peut être affiché ici. Veuillez vous reporter à l'édition en ligne http:// journals.openedition.org/itti/1349

JEAN-PAUL GÉHIN : Tes documentaires accordent une place importante, souvent essentielle aux entretiens. Ton travail documentaire propose un cinéma de la parole. Cependant, le statut de l'entretien et les formes des entretiens évoluent dans le temps et surtout suivant les thématiques abordées ou les personnes interviewées. Tu n'interviewes pas de la même manière, les journalistes vedettes ou les chercheurs. J'aimerais donc qu'on centre cet entretien sur la question de l'entretien, de la parole, de la fabrication de la parole en distinguant trois phases dans la production du documentaire : la phase de préparation de l'entretien, la phase de réalisation elle-même, la relation intervieweur/interviewé, la parole, et puis la phase de montage, l'organisation et la mise en scène de la parole.

\section{Je me repose beaucoup sur la parole, que ça soit celle de « l'ennemi » ou celle des « amis »}

Pierre Carles : Oui, je m'appuie beaucoup sur la parole mais il faut bien différencier le rapport à l'une et à l'autre. D'un côté, des entretiens avec des individus véhiculant des visions du monde minoritaires, marginales, porteurs de points de vue dissidents : ça peut être des chercheurs en sciences humaines comme Pierre Bourdieu et son équipe dans La Sociologie est un sport de combat, mais aussi des déserteurs de la guerre économique dans Attention danger travail, des individus engagés dans des modes de vie décroissant dans Volem rien foutre al païs, un incroyable mécréant comme le professeur Choron dans Choron dernière, d'anciens partisans de la lutte armée en France dans Ni vieux, ni traites... Dans ce cas-là, on s'ingénie à donner la possibilité à ces hommes et femmes dont on n'entend peu parler ou dont les discours sont peu audibles dans les grands médias quand ils ne sont pas caricaturés, de ne pas passer pour des hurluberlus, pour des dingues. Nous, nous les prenons au sérieux. L'intervieweur joue alors le rôle de passeur, d'intercesseur. C'est lui qui donne la possibilité au plus grand nombre de prendre connaissance de ces propos nonconventionnels, de ces pensées hétérodoxes.

Mais nous nous confrontons aussi dans mes documentaires à ceux qui détiennent le pouvoir, notamment le pouvoir médiatique, à ceux qui monopolisent la parole publique. Là, on a affaire à des responsables de l'information, à des gens qui bien souvent nous imposent une vision du monde, souvent la même, plutôt favorable aux politiques néo-libérales, complaisante avec l'hégémonie économique et militaire des 
Etats-Unis d'Amérique, plutôt méprisante à l'égard les mouvements sociaux, des politiques de nationalisations, etc... Et là, avec ces gens-là, l'entretien prend une autre tournure, la plupart du temps conflictuelle. On se retrouve, moi et les gens avec qui je travaille à mener un combat contre une parole hégémonique, contre les abus de pouvoir de responsables de l'information. Dès lors, tous les coups ou presque sont permis. Confrontés à des gens qui ne laissent guère de place à d'autres visions du monde que la leur, nous utilisons tous les moyens à notre disposition pour essayer de révéler leur vrai visage : ce sont d'abord et avant tout des hommes de pouvoir, nonélus mais prenant néanmoins la parole en public au nom du public. Ce n'est pas mal en soi d'être un homme de pouvoir ou un homme politique mais encore faudrait-il que ce soit clairement énoncé. Nous n'hésitons pas, à l'aide de méthodes parfois nonconventionnelles, à mettre en évidence les contradictions, les double-discours, parfois la mauvaise foi de ces hommes du pouvoir médiatique. Et nous ne nous privons pas de temps à autre de les railler, de nous moquer d'eux, d'abîmer leur image. Avec ceux qui contrôlent la parole dans les grands médias, il n'y a pas de scrupules à avoir. C'est ce que l'on peut observer dans Pas vu pas pris, Enfin pris ?, Fin de concession, mais aussi dans Hollande, DSK, etc ou, plus récemment, dans Les ânes ont soif.

\section{Le pouvoir d'évocation de la parole peut être très fort}

Multiplier les entretiens peut aussi être interprété comme une solution de facilité. Une telle place accordée à la parole peut signifier un échec: on est incapable de filmer le réel, résultat : on enregistre le discours des uns et des autres sur le réel. Il peut aussi y avoir des justifications économiques à cela : c'est moins cher de fabriquer des films où tu accordes cette large place à la parole. Cette dimension compte, bien entendu, il ne faut pas se leurrer.

Il y a un film qui m'a beaucoup marqué, en terme de place accordée à la parole, cela peut paraître étrange parce qu'à première vue il parait assez éloigné de mon univers, c'est le film de Jean Eustache Une sale histoire (1977, 28+22 minutes, les films du paradoxe) où Jean-Noël Picq et Michael Lonsdale racontent deux fois la même histoire. Ce qui est marquant dans ce film d'Eustache, c'est le pouvoir d'évocation de la parole. On voit à quel point certaines prises de parole peuvent littéralement produire des images. Je sais qu'Eustache avait pensé dans un premier temps illustrer le récit oral avant, finalement, d'y renoncer. Il avait imaginé plaquer des images de fiction sur l'histoire de voyeurisme narré par Jean-Noël Picq dans Une sale histoire mais il s'est vite aperçu qu'il n'arriverait pas à générer par ce procédé-là des images aussi fortes que celles suscitées par le récit du narrateur. J'ai toujours retenu cela dans un coin de ma tête : le pouvoir d'évocation de la parole, du moins dans certains cas. Un certain type de parole fabrique des images d'une rare puissance, tout comme la lecture de certains romans fabrique parfois de fortes images. La question est de savoir comment arriver à susciter ce genre de paroles, comment arriver à enregistrer ce type de propos qui donne la possibilité au spectateur de fabriquer de telles images.

Concernant l'autre type d'entretien, celui où l'on filme "l'ennemi", j'ai été très influencé par le travail de Marcel Ophuls, notamment dans Hôtel Terminus (documentaire, 1988, 287 minutes). Il interviewe des personnes qui, pendant la seconde guerre mondiale, étaient des collaborateurs ou des criminels de guerre. Il procède auprès de ces derniers sans dévoiler ses intentions, en faisant l'idiot la 
plupart du temps. Il y avait déjà fait cela dans un de ses premiers films, Le Chagrin et la pitié (documentaire, 1971, $251 \mathrm{mn}$ ). Ce n'est pas tant ce que disent ses interlocuteurs que leurs postures corporelles, le rictus de leur visage, leurs hésitations, leurs lapsus, leurs silences qui sont très parlants. Ce type d'entretien, j'ai sûrement essayé de le reproduire avec certains responsables de l'information que j'ai interviewés. Ce ne sont pas des criminels de guerre, mais des gens commettant des abus de pouvoir eux aussi...

\section{Le risque de l'empathie, c'est de se retrouver avec des entretiens pauvres}

Lorsque l'on filme "l'ami », il faut surmonter l'écueil de la trop grande proximité avec les gens filmés : tu les côtoies dans d'autres circonstances, tu as sympathisé avec eux, tu fais parfois partie du même milieu qu'eux et tu peux donc te retrouver avec des entretiens pauvres parce que tes interlocuteurs estiment que tu sais déjà beaucoup de choses et ne voient pas l'intérêt qu'il y aurait à te rappeler des choses que tu sais déjà, qu'ils t'ont déjà racontées. Voilà qui est parfois problématique dans ce type d'entretien.

Pour conjurer ce risque, notamment dans le film que j'ai consacré à Pierre Bourdieu, je n'ai pas réalisé d'entretien avec lui. Je me suis contenté de filmer toutes les situations où Bourdieu pouvait se trouver en mesure de développer ses analyses, devant des publics divers, notamment à l'étranger. Je m'étais rendu compte que quand il était confronté à des chercheurs ou même à des journalistes étrangers, il était amené à tenir pour non-acquises beaucoup plus de choses que face à un Français. Face à des interlocuteurs d'ici, il pouvait se montrer plus elliptique : « Mais ça vous le savez déjà, je ne vais quand même pas vous le redire ». Pour éviter cet écueil, je me suis fait le plus petit possible, je suis mis sur l'épaule de Pierre Bourdieu et je l'ai accompagné partout où je pouvais avec l'objectif de filmer des moments où il serait amené à reformuler ses analyses, face à des publics de non-sociologues parfois.

Sur d'autres films, où je n'ai pas eu cette possibilité-là, je me suis rabattu sur des entretiens plus classiques, plus conventionnels. Je pense au travail que j'ai fait sur Action directe avec Georges Minangoy (Ni vieux, ni traîtres, 2006). Nous avons donné la parole à des gens considérés comme des terroristes par le système médiatique. Or notre objectif était que le public puisse entendre un autre son de cloche sur les actions d'Action directe que ce que l'on entend habituellement : « ce n'est pas bien de poser des bombes ", «ce n'est pas bien de tuer un patron ", "c'est ultra-violent ", etc... Je précise que, lorsque quelqu'un se rend dans une salle de cinéma pour voir un film, il a en tête le discours ambiant, il n'est pas neutre. Il a dans son cerveau, des idées reçues, des images déjà toutes fabriquées, des représentations dominantes... C'est la raison pour laquelle dans ce film nous ne donnons pas la parole à ceux qui vont rappeler qu'un attentat à l'explosif, ce n'est pas bien ou qu'assassiner un PDG, c'est très mal. Le spectateur le sait déjà lorsqu'il rentre dans la salle de cinéma, inutile de le lui rappeler. Les rapports de force ne disparaissent pas subitement à l'entrée des salles. Nous sommes traversés par ces rapports de force lorsque nous voyons un film. Certaines idées sont déjà incrustées dans notre tête, d'autres non. Certains points de vue sont hégémoniques, d'autres pas. Notre boulot, à nous 
réalisateurs indépendants, c'est d'aider à faire entendre ceux que l'on entend peu ou mal.

\section{Dans mes films, on ne donne pas la parole à ceux qu'on entend déjà tout le temps}

Ce sont des paroles de dominés. Bon, on va me dire que Bourdieu détenait un grand pouvoir dans le cercle académique parce qu'il était professeur au Collège de France, le poste le plus prestigieux pour un savant dans ce pays. Mais dans le champ médiatique, il n'était pas dominant. Ses analyses n'étaient pas hégémoniques, loin de là. Lorsque j'ai réalisé le film sur lui, peu de temps après ses prises de paroles publiques pro-gréviste lors du mouvement social de 1995, la télévision et la radio le présentaient comme un dangereux gauchiste. Ce sont ces adversaires qui monopolisaient la parole dans l'espace public, pas lui. D'où le parti pris de ne pas donner la parole à ses contradicteurs dans La Sociologie est un sport de combat. Ceux qui dénigraient les analyses de Bourdieu avaient tout loisir de s'exprimer où ils le souhaitaient, notamment dans les grands médias. Il me semblait que ce déséquilibre visait à compenser, autant que faire se peut, le déficit de point de vue «bourdieusien » dans le champ médiatique. Ce parti-pris transparait dans tous mes films.

Quand on laisse s'exprimer les dominants, c'est plutôt pour les ridiculiser. Quand on donne la parole, en revanche, à des individus n'étant pas en situation d'hégémonie intellectuelle, c'est plutôt pour les aider à exposer leur point de vue dans de bonnes conditions. Dans Les ânes ont soif, Patrick Bèle, Maurice Lemoine et Mylène Sauloy, dont les propos vont à l'encontre du discours dominant, prennent la parole dans de bonnes conditions pour raconter ce qui se passe en Equateur. Dans nos grands médias, Hugo Chavez, qui a gagné près d'une dizaine d'élections, s'est fait traiter de dictateur, comme on a pu l'entendre sur ARTE. Nous n'allons pas colporter ce genre d'imbécilités. Ce qui ne veut pas dire que nous ne serons pas critiques à l'encontre de la politique de Rafael Correa lorsque nous tournerons la suite du film en Equateur. Làbas, sur place, Rafael Correa a la possibilité de se faire entendre, sa parole n'est pas marginalisée comme elle l'est ici. Rien ne nous empêchera donc de porter un critique sur sa politique.

JPG : Pour en revenir aux deux types d'entretien, ce qui me frappe c'est que tu appliques les techniques des journalistes pour les journalistes, et les entretiens approfondis de recherche et d'écoute, pour les chercheurs.

\section{Retourner leurs méthodes aux journalistes dominants}

PC : Oui, c'est vrai que confrontés à des journalistes qui sont la plupart du temps des responsables de l'information et non pas des prolétaires de ce métier-là, nous nous amusons à leur retourner leurs méthodes, à faire preuve de la même noncomplaisance avec le pouvoir que celle qu'ils revendiquent, que celle qu'ils affichent en public. Ces responsables de l'information, qui sont des hommes de pouvoir, mettent souvent en avant leur qualité de journaliste pour éviter d'apparaitre comme des dominants. Or en tant que journalistes, ils se doivent de revendiquer des méthodes d'interview sans complaisance, «à l'américaine " ou « à l'anglo-saxonne ", 
largement mythifiées, précisons-le au passage et essaient de se faire passer pour des gens jouant le rôle de contre-pouvoir à l'égard des puissants. À les écouter, ils feraient des enquêtes indépendantes et des interviews sans concession des dirigeants politiques ou économiques... Aussi, ils n'apprécient guère qu'on leur applique ces mêmes méthodes. Ils trouvent cela non-confraternel. On n'hésite donc pas à les interrompre lorsqu'ils profèrent des contre-vérités, à leur mettre des documents embarrassants sous les yeux si cela se justifie, à leur poser des questions sans leur avoir transmis les questions au préalable. Ils se retrouvent pris dans des dispositifs d'interviews qui ne leur donnent pas le pouvoir, pour une fois. Ça ne leur plait que moyennement. Pour réussir à les filmer, pour nous introduire dans leurs bureaux avec une caméra, nous laissons entendre que nous venons leur cirer les pompes. Nous flattons leur égo en leur mettant dans les pattes des journalistes étrangers qui leur font les yeux doux, des complices qui leur disent qu'en Belgique ou qu'en Uruguay on adore leur travail. Ils tombent dans le panneau la plupart du temps. Lors de l'entretien, ils ne peuvent s'empêcher de faire leur autopromotion. Ils lâchent des propos complaisants à l'égard de leur travail et c'est parfois cela qui se révèle terrible pour eux. Ils ne se rendent pas toujours compte à quel point ils se dévoilent, à quel point ils laissent paraître leur vision du monde. C'est ensuite, au montage, en juxtaposant leurs propos avec d'autres interviews ou archives qu'apparaîtra le caractère extrêmement partisan ou orienté de leur propos. Dans les deux cas de figure, nous allons à leur rencontre pour leur faire la guerre ou plutôt la guérilla puisque nos méthodes sont des méthodes non-conventionnelles. Nous pourrions reprendre la devise des guérilleros : «mords et fuis ». Mais nous n'agissons pas ainsi, bien entendu, avec des gens à qui nous souhaitons donner la possibilité d'exprimer des points de vue dissidents.

JPG : Faut-il toujours voir tes documentaires par rapport aux dominants, par rapport aux paroles en situation d'hégémonie?

PC : Oui. Quand on fait des films, on les fait «pour» mais aussi «contre». Pour mettre en avant certaines pensées et contre la pensée dominante. Il y a aussi du pour et du contre à un autre niveau: on fait des films "contre» d'autres réalisateurs, contre nos concurrents, pour se démarquer d'eux. Ce n'est pas toujours pour des raisons très nobles. Il faut percevoir tout ce que l'on fait à la lueur de ce que font aussi les autres. C'est ce qui se passe dans tous les domaines : pour savoir pourquoi quelqu'un fait quelque chose, il faut observer ce que font les autres, du moins ceux qui travaillent dans le même univers, dans le même champ pour reprendre l'expression de Bourdieu. Il a analysé cela dans un de ces derniers cours au collège de France sur le peintre Manet.

Mon principal souci, en tant que réalisateur, c'est de donner la possibilité d'entendre ces sons de cloches minoritaires, hérétiques, dissidents, non orthodoxes. Et ça passe parfois par le fait de donner la parole aux ennemis de mes ennemis. Donner la parole à des gens qui dans leur domaine combattent aussi les mêmes cibles que les miennes. Alors, ça serait quelque chose d'insupportable si les gens qui figurent dans ces films se retrouvaient tout à coup dans des positions dominantes, d'hégémonie. Mais on en est loin. Si le discours des anciens d'Action directe, justifiant la lutte armée, devenait hégémonique dans ce pays, ce serait tout aussi insupportable. Ces paroles ne m'intéressent que dans la mesure où elles sont minoritaires. Tout cela pour dire qu'il 
n'est pas possible d'isoler mes films du cadre dans lequel ils s'inscrivent, celui du champ médiatique et, plus généralement, des rapports de force dans l'espace public.

JPG : Pourquoi ce cinéma qui souhaite s'attaquer aux dominants cible rarement le pouvoir économique? Je me rappelle de Pierre Carles plus jeune (Attention danger travail, réalisé avec Christophe Coello et Stéphane Goxe, 2003), interviewant le patron des patrons.

\section{Les grands médias jouent le rôle de bras armé du patronat}

PC : C'est une erreur, me semble-t-il, de dissocier le patronat du système médiatique. Ce dernier n'est-il pas l'allié des grands patrons quand ceux-ci ne dirigent pas carrément certains médias? Si une oligarchie se maintient au pouvoir, se reproduit, c'est en grande partie grâce au rôle joué par ces grands médias qui naturalisent les inégalités sociales, qui les font passer pour normales ou inévitables. Ce système médiatique joue aussi le rôle d'entreprise de résignation : "N'imaginez pas que l'on puisse gouverner autrement ou s'organiser autrement en société que comme nous le faisons actuellement». Si beaucoup de gens pensent aujourd'hui qu'il n'y a pas d'autre monde possible, c'est parce que ces médias font un boulot de fossoyeurs d'utopies, incitent les gens à accepter leur sort alors qu'il est peu enviable pour bon nombre d'habitants de cette planète. Bref, la critique des médias c'est une critique du pouvoir économique et, partant, du système capitaliste.

JPG : Pour toi, l'entrée principale de la lutte contre le néolibéralisme, c'est montrer les médias, tels qu'ils sont, les grands médias tels qu'ils sont?

PC : Je n'ai pas dit que c'était la porte d'entrée principale mais c'en est une. Et je ne suis pas le seul à le penser. Il y a d'autres réalisateurs, d'autres journalistes indépendants, essayistes, écrivains, chercheurs en sciences humaines, qui cherchent à dévoiler les relations de domination, qui tentent de mettre en évidence les mécanismes de lutte des classes. C'est un boulot que font d'autres personnes, depuis bien longtemps. Il s'avère que je suis devenu un des spécialistes français du système médiatique à force de travailler sur ce sujet. J'ai réalisé plusieurs films sur ce thème à l'époque où pas grand monde ne s'y intéressait. Je n'étais pas forcément un grand réalisateur mais comme personne ne s'intéressait à ces choses-là à l'époque, que ce soit sur le petit écran ou au cinéma, j'ai fait ce boulot-là : un travail de critique des médias. Et s'il a eu une certaine audience, c'est peut-être tout simplement parce qu'il n'y avait pas grand monde sur ce terrain-là au début des années 1990. Il existait des livres, on commençait à organiser des colloques, mais dans le domaine audiovisuel, rien ou pas grand-chose. S'ajoutait aussi le fait que je venais de la télévision, que j'y avais travaillé, ce qui donnait plus de poids à mon travail critique. C'est de l'intérieur, en quelque sorte, que j'opérais cette critique.

Mon premier film, Pas vu pas pris, a eu un certain succès en salles de cinéma, notamment parce qu'on y voyait un type, moi, en l'occurrence, travaillant à la télévision et racontant ce qu'il y avait observé de l'intérieur, chose qui n'avait pas été trop faite jusque-là. Le personnage que j'incarnais à l'écran trahissait d'une certaine manière son milieu. Si ça avait été simplement un pamphlet ou la critique d'un observateur extérieur, ça n'aurait pas eu la même force. D'ailleurs, les réactions ont été proportionnelles à cette trahison. Bon, maintenant, si ce premier film avait fait un bide, peut-être ne serais-je pas en train de poursuivre ce travail aujourd'hui. C'est 
probablement parce qu'il y avait des profits, notamment symboliques, à réaliser ce travail critique à l'égard des médias que j'ai persévéré. Certains le voient comme un sacrifice de ma part parce que je me suis grillé avec la télévision en faisant cela. Mais n'oublions pas la phrase de Bourdieu : « on a intérêt au désintéressement ».

JPG : La première phase de réalisation de documentaire, c'est la phase de préenquête, la phase de préparation de l'entretien. Tu peux nous décrire comment tu fais. Prépares-tu tes entretiens ? De quelle manière ? Vas-tu sur le terrain sans la caméra?

PC : Quand tu réalises les entretiens avec ceux que l'on pourrait appeler les « amis », tu convoques tout un tas d'expériences, de savoirs. Il existe aussi des intervieweurs qui débarquent sans préparation et qui reviennent avec des résultats formidables mais ça reste exceptionnel. Moi, je suis plutôt quelqu'un de laborieux. Les gens que je vais voir, je les rencontre souvent avant, je me suis documenté sur ce qu'ils font ou ont fait, j'essaie de deviner à peu près dans quelle direction il serait judicieux d'orienter l'entretien. Quand je pars filmer Bourdieu, je n'ai pas découvert son travail quelques jours plus tôt. Depuis de longues années, je me suis retrouvé, d'abord à la fac en tant qu'étudiant en sociologie, puis par certaines lectures ou rencontres, à m'intéresser à la sociologie critique développée par Pierre Bourdieu. Même chose pour le professeur Choron.

Après, ce sont souvent des opportunités, qui font le larron, les hasards de l'existence qui débouchent sur le tournage d'un film. Je n'aurais pas pu faire un film sur Pierre Bourdieu si je n'avais pas réalisé un film de critique des médias (Pas vu pas pris) repéré par ce dernier. C'est après l'avoir vu, qu'il ne s'est pas montré trop hostile à ce que je lui consacre un film. Même chose pour Action directe. Avec Georges Minangoy, nous avons rencontré un type qui les connaissait bien, qui allait les visiter en prison. De fil en aiguille, nous nous sommes retrouvés embarqué dans le tournage de $\mathrm{Ni}$ vieux, $n i$ traîtres. Ce n'est pas nous qui avons décidé. Nous avons été instrumentalisés en quelque sorte. Mais pour la bonne cause.

\section{II faut donner quelques gages d'indépendance}

Et l'indépendance ce n'est pas simplement l'indépendance d'esprit. C'est l'indépendance économique pour commencer. C'est ça la première chose. Impossible de filmer ces personnes-là, si tu n'as pas fourni la preuve que tu pouvais fabriquer des objets audiovisuels avec un certain degré d'indépendance, sans être soumis au bon vouloir ou au contrôle du petit écran ou parfois même des institutions finançant l'audiovisuel. On te fera plus confiance si tu es un réalisateur indépendant que si tu annonces que tu travailles pour TF1, France 2, M6 et même ARTE. Sans parler des financements institutionnels. Quand tu connais le point de vue des institutions sur la lutte armée, par exemple... Sans donner de tels gages d'indépendance, les gens que tu veux filmer seront très méfiants à ton égard. Ce qui ne nous a pas empêchés de faire des mécontents chez les gens se disant proches d'Action directe. Mais là c'était pour d'autres raisons, en raison de dissensions internes : si untel s'exprimait dans le film, tel autre ne voudrait pas apparaître, ce genre de choses.

Une fois que tu arrives à entrer en contact avec des gens aux discours plutôt radicaux et qui ont commis des actes illégaux, il faut trouver le bon endroit, la bonne distance. Etre suffisamment près d'eux pour les mettre en confiance mais pas trop près, non plus, sinon ils estiment que tout un tas de choses sont déjà acquises, sont connues du 
public. J'ai entamé un travail sur la guérilla des FARC (Forces armées Révolutionnaires de Colombie), le principal mouvement de lutte armée d'Amérique. On a réalisé des entretiens avec des guérilleros ayant des années de résistance derrière eux, qui ont passé des années dans le maquis. En tant que passeur, je dois livrer des informations à un public européen qui ne sait rien ou presque sur les FARC à l'exception du fait que ce sont de "méchants terroristes" qui ont séquestré la "gentille Ingrid Betancourt». Or, ce que je recherche, c'est de remettre ces guérilleros à leur place dans l'histoire de la résistance ou des résistances. Parce que ce sont avant tout des résistants et non pas des narcotrafiquants comme le racontent la plupart des grands médias et, bien entendu, les gouvernements de Colombie et des USA. Il se peut que certains aient trempé dans le trafic de cocaïne à des fins d'enrichissement personnel ou participé à des crimes de guerre comme chaque fois qu'il y a un conflit armé mais ça tient de l'exception. Pour en revenir aux entretiens avec ces gens-là, il n'y a pas de recette : il faut s'en rapprocher tout en n'étant pas trop proche.

JPG : Certes, il n'y a a pas de recette mais toi comment tu fais ? C'est-à-dire, est-ce que tu prépares tes questions? Est-ce que tu viens sans caméra d'abord?

PC : Tout dépend des interlocuteurs. Avec les FARC, si tu viens sans caméra, ils vont se demander pourquoi. Ils sont prêts à te recevoir, à donner de leur temps mais ils restent méfiants. Donc si tu viens sans caméra, c'est un peu suspect. Ils se demandent si tu n'es pas membre d'un service de renseignement... Il vaut mieux, pour les rassurer, venir avec une caméra. Il n'y a pas de règle en la matière. C'est au cas par cas.

La moindre de choses, c'est d'arriver avec un peu de background, de connaissances du sujet, de s'être au préalable documenté. Et pendant l'entretien, il faut tenter d'oublier cette connaissance que tu peux avoir des problèmes qui vont être abordées, essayer de retrouver une position "naïve ». Quand on fait des entretiens préparatoires, car ça m'arrive parfois de rencontrer les gens sans caméra avant le jour du tournage, il vaut mieux laisser s'écouler un peu de temps avant de revenir les voir. Quand tu retournes les filmer, en laissant passer un certain temps, ils ont souvent oublié ce qu'ils t'avaient raconté la première fois. De plus, tu reviens avec une équipe, un ingénieur du son, un caméraman, même s'ils se souviennent de ce qu'ils t'ont raconté, il suffit de leur dire: "les membres de l'équipe ne sont pas courant de ce que tu m'avais raconté la fois dernière » et les voilà obligés de reformuler ce qu'ils t'avaient dit.

Voilà quelques « recettes ». Il faut arriver bien-sûr avec une connaissance du sujet, parce que si ton interlocuteur s'aperçoit que tu ne sais pas grand-chose, il va se refermer, ça risque de mal se passer. Mais attention de ne pas être vu comme trop pointu sur la question car il y a le risque qu'il se dise aussi « ça il le sait déjà, je ne vais pas le lui raconter ».

\section{Plus que des questions, ce sont des dispositifs qui sont préparés}


JPG : Et dans ton rôle de cinéaste, quand tu fais des entretiens, avec «l'ennemi » disons, tu prépares des questions, tu as des questions qui permettent de produire un effet, de produire une parole?

PC : Il n'y a pas de règle. Ça m'est arrivé de fabriquer des impostures pour accéder à certaines personnes. Dans Fin de concession, nous cherchions à interpeller l'ancien numéro 2 de TF1, Etienne Mougeotte, qui était devenu directeur du Figaro. Nous voulions essayer de le coincer sur l'histoire de la concession de TF1, lui faire admettre certaines choses. Si on lui avait demandé une interview en ne dissimulant rien de nos intentions, il n'aurait pas donné suite à notre demande d'entretien. On s'est donc fait passer pour une équipe latino-américaine. J'étais censé incarné un caméraman uruguayen qui accompagnait une vraie journaliste uruguayenne, Virginia Martinez. Et Etienne Mougeotte, comme beaucoup d'hommes de pouvoirs, à un égo tellement surdimensionné, qu'il ne ne s'est pas douté qu'on n'en avait rien à foutre de lui en Uruguay. Il pensait qu'on s'intéressait à lui d'aussi loin. L'interview n'a pas donné les résultats escomptés mais ça c'est une autre histoire.

La plupart de nos "ennemis " sont des gens qui ne soupçonnent pas qu'on puisse venir avec des intentions de mettre en évidence leurs contradictions ou une certaine malhonnêteté. Ils sont tellement habitués à des entretiens complaisants, à des interviews " cirages de pompes ", qu'ils sont rarement sur leurs gardes lorsqu'on les rencontre. Surtout quand une journaliste belge, comme Aurore Van Opstal qui apparait dans mes deux derniers films, va les voir. Ils ne soupçonnent pas une seconde que cette jeune journaliste belge pourrait présenter un danger pour eux.

Quand j'ai réalisé Pas vu pas pris, ces responsables de l'information ont cru avoir à faire à l'époque à un jeune journaliste débutant, un peu naïf, qu'ils allaient retourner comme une crêpe. Ils n'imaginaient pas que j'allais leur sortir un petit magnétoscope et leur montrer des images taboues sur le petit écran qui allaient les conduites à raconter un peu n'importe quoi. Tout cela était préparé, rien n'était improvisé. Le dispositif avait été conçu bien avant l'entretien et le piège allait se refermer sur eux une fois que l'on aurait mis le pied dans leur bureau. Ils se retrouvaient sommés de s'expliquer sur la censure à la télévision française alors qu'ils avaient prétendu, quelques minutes plus tard qu'elle n'existait pas. Une fois que la caméra tournait, c'était fichu pour eux. En tant qu'hommes de pouvoir, soucieux de leur image, ils savaient bien, qu'une interruption d'interview sous l'oeil de la caméra risquait d'être quelque chose de ravageur pour eux. Ils mettaient un peu de temps avant de comprendre que le dispositif d'entretien, pour une fois, n'était pas franchement en leur faveur. Ils s'en apercevaient trop tard et ils n'avaient pas 10000 solutions : soit ils interrompaient l'entretien, c'est arrivé mais quand quelqu'un interrompt un entretien, cela suppose qu'il y a quelque chose à cacher ce qui n'est pas terrible pour son image de journaliste, ou bien l'entretien se poursuivait, ce qui nous permettait d'avancer des preuves supplémentaires de leur mauvaise foi, de leur double langage, de leur hypocrisie. Ils s'enferraient dans leurs explications et en sortaient rarement gagnants.

JPG : Donc plutôt préparer un dispositif que des questions parce que ça renvoie au produit fini.

PC : Ces dispositifs d'interviews, où les puissants ne sont pas maitres de la situation pour une fois, peuvent constituer une sorte de revanche pour les spectateurs. Ils se projettent dans quelqu'un qui va affronter un homme de pouvoir commettant un 
abus de pouvoir. Il y a un plaisir un peu sadique à voir ces puissants malmenés. Les spectateurs peuvent se sentir vengés. C'est un dispositif narratif, que je n'ai pas inventé. J'ai simplement introduit sur le terrain de la critique des médias des méthodes qui avaient été mises au point, par exemple, par Marcel Ophuls ou remises au goût du jour par Michael Moore

JPG : Je voulais revenir sur la question de l'entretien, c'est aussi une mise en scène ces entretiens filmés de la relation entre l'intervieweur et l'interviewé.

PC : On ne peut pas faire abstraction de la caméra. C'est un spectacle et cela introduit des limites à l'entretien. Quand tu filmes «l'ennemi », ce que tu recherches en tant que réalisateur, c'est le moment où le puissant va montrer des signes de faiblesse, ce moment où il va tomber de son piédestal, où il ne sera plus en mesure de se servir de son pouvoir pour asseoir son autorité.

Maintenant, ne nous leurrons pas: quand tu filmes l' "ami», tu joues aussi la comédie, tu fais du cinéma et lui aussi fait du cinéma, toujours à cause de la présence de la caméra. Tu te retrouves rarement dans la situation d'apprendre quelque chose de ton interlocuteur sur le moment. Tu as toujours une pensée pour le spectateur. Que va-t-il apprendre que tu sais déjà. Ce dernier est au-dessus de toi, ou perché sur ton épaule. Tu n'es que l'interface, le passeur. L'objectif est de faire en sorte que quelque chose circule. Si tu as réussi à permettre au spectateur de prendre connaissance de propos subversifs, d'idées non-reçues, c'est pas mal. Mais c'est sûrement très frustrant pour un vrai spécialiste du sujet abordé lors de l'entretien d'entendre ce qui se dit. Ça reste malgré tout superficiel. C'est plutôt ce que j'appellerai une mise en appétit. Je ne cherche pas me substituer au travail du spécialiste ou du vulgarisateur mais essayer plutôt de donner envie de se documenter, de faire un bout de chemin avec tel ou tel personne incarnant telle ou telle pensée ou telle ou telle pratique qui me semble digne d'intérêt. Voilà, mon boulot : mettre en appétit.

\section{Construire une parole qui devient collective}

JPG : Ca a un côté assez frustrant : avoir des entretiens approfondis et n'en monter que quelques minutes.

PC : Bien-sûr. Et tu ne te sens pas toujours très bien vis-à-vis des gens qui t'ont consacré une bonne partie de leur temps, qui ont parfois mis beaucoup d'eux dans l'entretien. A l'arrivée, ça peut être très frustrant. Maintenant, il faut se dire que même si telle ou telle personne ne s'exprime que quatre ou cinq minutes dans un documentaire, sa parole va aussi s'appuyer sur celle de ces voisins dans le film. On ne peut pas la considérer comme isolée ou détachée de celles des autres. Ce n'est pas ta faible présence temporelle qui préjuge de la présence intellectuelle. Tu peux être peu présent dans un film en termes de durée, avoir une séquence courte et, en même temps, être très présent parce que ton intervention éclaire ou renforce celle des autres et va prendre une ampleur insoupçonnée.

Il peut y avoir des frustrations de la part des personnes interviewés ou des spectateurs, mais ce n'est pas si grave finalement. C'est déjà mieux que rien. Prenons le film que j'ai réalisé avec Georges Minangoy sur le groupe armé Action directe. Il n'y avait pas, à l'époque, de film sur Action directe en dehors de reportages télévisés présentant les membres d'AD comme des dingues, des fous furieux. Même si Ni vieux, 
ni traitres a de fortes insuffisances, c'est mieux que rien de casser cette représentation là en les présentant comme des gens sensés, leurs attentats comme des actions réfléchies. Sur Action directe ou sur les FARC, des mouvements de lutte armée, il y a eu un tel travail de dénigrement, une telle diabolisation de ce type de combat par les grands médias que leur parole est quasiment inaudible aujourd'hui.

JPG : Comment faire pour rendre audible ce type de discours?

PC : Il faut commencer par présenter ces gens qui ont mené ce type de combat comme des êtres humains et non pas comme des monstres. Il faut essayer de casser ces représentations dominantes et, une fois qu'elles sont ébréchées, réussir à mettre le spectateur en condition d'entendre quelque chose qu'il estimait insupportable, ne serait-ce que parce que la figure du terroriste est devenue la figure moderne du diable. Il faut rappeler, en ce qui concerne le terrorisme, que ce n'est pas la même chose de commettre un attentat ciblé et de tuer des gens plus ou moins au hasard lors d'une action aveugle. Rappelez aussi que ceux que l'on qualifie de terroristes à une époque peuvent du jour au lendemain devenir des gens fréquentables si un accord de paix est signé avec leur organisation, comme ce fut le cas avec les membres de l'IRA (Irish Republican Army) en Irlande du nord. Des gens qui étaient jusque-là désignés comme des terroristes par l'armée britannique et les grands médias sont devenus du jour au lendemain d'anciens résistants. Ces petits rappels historiques permettent parfois d'aider à relativiser certains propos.

JPG : Pour revenir sur le rôle de passeur, comment tu mets en scène l'intervieweur? Parce que dans pas mal de tes films l'intervieweur il est là, il est présent, en voix-off ou même devant la caméra.

\section{Défier le récit des puissants}

PC : De toute façon, même hors champ, il est toujours là l'intervieweur. La personne filmée s'adresse forcément à un interlocuteur, qu'on l'aperçoive ou pas. Il y a toujours quelqu'un qui est présent, hors champ, même s'il n'est pas visible ou audible. Dans certains de mes films, l'intervieweur est assez présent à l'image, dans d'autres non. Je n'ai pas de règle en la matière, c'est au montage qu'on décide ou pas de garder certains propos et un certain degré de présence. Je n'ai pas vraiment travaillé cette question.

Quand je filme «l'ennemi », l'intervieweur est plus souvent présent parce que c'est pour le spectateur, la figure du vengeur. Il y a un plaisir à voir, notamment physiquement, cette personne mettre à mal les puissants. Tout cela me rappelle aussi une phrase de Ken Loach qui donne son titre à un petit bouquin édité récemment : « Défier le récit des puissants » (2014, 48p. Indigène éditions). C'est cela notre boulot : défier le récit de ces gens-là. Mais comme on fait de l'audiovisuel et non de l'écrit, tout cela doit être incarné. C'est en allant à la rencontre de leur corps, dans leur bureau, dans leur univers professionnel, que l'on cherche à contester leur récit. Dans Les ânes ont soif, le premier épisode d'Opération Correa, quand tu vois Julien Brygo jouer les sales garnements, rentrer dans le bureau d'Yves Calvi, lui reposer dix fois la même question, ça participe aussi au plaisir que l'on peut avoir en tant que spectateur d'agir par procuration. On voit cet homme de pouvoir interpellé par quelqu'un qu'il n'a pas convié chez lui, s'inviter dans ses bureaux, mettre le pied dans la porte, se faire virer par la porte pour rentrer par la fenêtre... Enfin il y a ce plaisir de voir que Calvi n'a 
pas eu le temps de mettre en place son système de communication, de langue de bois habituel. Il est pris au dépourvu lorsqu'il est confronté à un véritable arapède comme Julien Brygo. Ce dernier représente la figure du scotch que le capitaine Haddock dans Tintin n'arrive pas à décoller de son doigt. Et pour le spectateur, il y a le plaisir de s'identifier à David contre Goliath.

JPG : I'intervieweur est aussi un comédien. C'est d'ailleurs vrai dans d'autres films. C'était toi dans Pas vu pas pris.

L'idéal est de conduire son interlocuteur à formuler quelque chose à un moment donné qu'il n'avait jamais formulé de telle manière. C'est la magie du documentaire.

PC : Dans Pas vu pas pris, c'est moi en effet qui incarnait cette figure d'arapède. Puis d'autres ont pris le relais dans mes derniers films. L'objectif lors d'un entretien filmé, c'est de fabriquer un moment unique, non reproductible. Idéalement, il faudrait que la caméra ait enregistré quelque chose qui se serait passée entre toi et l'interviewé, inscrit dans l'instant. Là, on est dans le documentaire. La caméra a saisi ou généré quelque chose de rate à ce moment-là. C'est pas forcément quelque chose qui n'a jamais été dit, ça peut-être quelque chose qui n'aurait jamais été formulé de telle manière. Mais toi, par ton travail, par ta présence, tu as conduit untel à formuler telle réflexion, telle pensée sensible, ce jour-là. Ça, c'est le grand plaisir que tu peux avoir en tant qu'intervieweur. Et peu importe la justesse du cadre, la qualité de l'image, il y a une vérité documentaire qui surgit, il se passe quelque chose qui ne s'était pas produit avant et qui ne se reproduira pas après. Ce que fait rarement la télévision : guetter ce moment-là. Le reportage télévisé ou ce qu'on appelle « documentaire » à la télé, c'est plutôt de la fabrication en série, des objets audiovisuels fabriqués à la chaîne, de l'industrie alors que le documentaire est de l'ordre du prototype, de la fabrication artisanale. L'objectif c'est de fabriquer un film qui soit un prototype, qui soit un objet unique et non pas une série.

C'est l'objectif visé mais c'est difficile... Faire émerger une parole singulière parce que tu as conduit ton interlocuteur dans une relation de confiance qui lui a permis de dire quelque chose qu'il n'avait pas pu exprimer jusque-là. Plus qu'un métier de passeur, c'est un métier d'accoucheur. C'est très rare d'y parvenir. J'ai rarement réussi cela mais je sais que c'est ce que je vise en tant qu'intervieweur. Cela peut surgir lors de l'entretien préparatoire, lorsque la caméra n'est pas présente, au téléphone ou lors d'interactions qui ne sont pas enregistrées. Le bonheur c'est quand ça se déroule sous l'oeil de la caméra, dans de bonnes conditions techniques, avec un son correct, une image pas trop dégueulasse... Je pense que la vérité documentaire est tellement forte, qu'à partir du moment qu'il y a du son et de l'image, je dirai presque que la qualité de l'image - ce n'est pas la même chose avec le son - est secondaire. Le spectateur s'en fiche, il voit que ce qui se déroule à ce moment-là est quelque chose de très singulier.

JPG : Justement, l'émergence d'une parole singulière, ce qu'on recherche j'allais dire tous. En tout cas toi, parce que c'est une coproduction intervieweur interviewé, est-ce que c'est pour ça que l'intervieweur est très présent dans ton cinéma?

PC : Est ce qu'il est très présent ? Oui et non...Dans La Sociologie est un sport de combat, le filmeur est absent à l'image et au son, il est vrai qu'il ne fait pas d'interview. Il y a juste un moment où Bourdieu lisant une lettre incompréhensible de Jean-Luc Godard m'interpelle. Il me demande mon avis.. Mais c'est l'une des rares références au filmeur... Dans ce qu'on a montré ici lors de la dernière édition de Filmer le travail, un premier montage des rushes de Bourdieu le retour, réalisé avec Annie Gonzalez, le 
dispositif est différent mais nos interventions peu présentes. Lorsqu'on filme l' " ennemi », oui, nos corps sont présents, mais lorsqu'on filme l'ami ils s'effacent. On désire mettre en valeur ses paroles et je ne crois pas que ce soit nécessaire que l'intervieweur soit présent physiquement ni même phoniquement. On essaie plutôt de se concentrer sur cette parole singulière.

JPG : Moi j'avais le sentiment que tu trouvais importante cette coproduction et que tu la mettais en scène. Je pense au film sur les étangs de Bages-Sigean.

\section{Cela m'intéresse d'expérimenter, de tenter des choses ; j'ai pas la science infuse, donc je tâtonne, j'essaye...}

PC : Là c'est différent. Avec Philippe Lespinasse, le co-réalisateur de Gruissan à la voile et à la rame, nous ne nous attardions pas avec les gens que nous filmions. Il s'agissait de rencontres relativement brèves avec des habitants du bord des étangs de la méditerranée, des gens que nous ne croisions que pendant quelques heures. Nous nous déplacions en canoë kayak dans ce monde rural et aquatique sachant que nous, les filmeurs, sommes des urbains, peu familiers de la pêche, de la chasse, de la cueillette... Notre mode de vie est à des années-lumière du celui que nous filmions. Nous avons choisi de montrer cette dichotomie. Nous avons mis en scène notre parcours afin que le spectateur sache d'où nous parlions. L'envers du décor du tournage était donné à voir y compris nos bisbilles de réalisateurs qui peuvent paraître parfois un peu éloignées des préoccupations des gens que nous filmions... Tu as raison, on est présent, mais parce que le film intègre son propre making of en quelque sorte. C'est un film où le travail des filmeurs, leurs divergences de vues sont portées à la connaissance des spectateurs. On voulait voir ce que ça donnait. C'est la raison pour laquelle, nous sommes relativement présents, Philippe Lespinasse et moi dans Gruissan à la voile et à la rame... D'ailleurs, je ne suis pas sûr que le dosage entre notre histoire et la leur soit le bon, qu'on ait réussi notre coup. On n'évite pas toujours l'écueil du narcissisme.

Ça marche, ça marche pas...ce qui est sûr c'est que je ne dissimule pas le dispositif filmique. Je ne cache pas trop d'où je parle. Quels que soient les sujets abordés, il me semble important que le spectateur sache d'où on parle. Cela peut passer parfois par la présence physique, ou les tutoiements par exemple. Quand je tutoie quelqu'un dans un film, je ne le cache pas, nous le gardons au montage. S'il y a cette relation de proximité avec les filmés, elle n'est pas dissimulée au spectateur. Ces éléments-là, nous essayons de ne pas les gommer.

JPG : Dans le film sur les travailleurs de l'étang de Bages-Sigean, il y a donc le travail des filmeurs mais aussi, ça va peut-être faire la transition avec la troisième partie, sur le montage, une leçon de montage.

PC : Oui, il y a une vraie-fausse leçon de montage. C'est un simulacre de montage, parce que le monteur qui apparaît dans le film, comme personnage du film donc, Roger Ikhlef, travaille sur un film qui... n'est pas celui que nous sommes en train de voir. Nous lui avions fourni les images du premier montage de Gruissan à la voile et à la rame mais ce n'est pas lui qui a monté les séquences dans lequel on le voit devant le banc de montage. Toi, spectateur, tu as l'illusion que c'est le cas, que c'est le monteur de la totalité du film. Une des règles d'or de ce monteur, qui est décédé, c'était de ne 
pas monter d'images auxquelles on est attaché affectivement, notamment celles dans lesquelles on est présent à l'image ou même hors champ. Or, dans Gruissan à la voile et à la rame il y a l'illusion que le personnage qui monte des bouts de notre canoë-movie est le monteur du film complet. C'est une illusion. Comme dans Enfin pris où figure une séquence de psychanalyse. Mais c'est un simulacre d'analyse car, par définition, il n'y a pas de tiers présents lors d'une séance d'analyse, a fortiori une caméra. Attention de ne pas se faire abuser par ces mises en abyme. En ce qui concerne Roger Ikhlef, je trouvais aussi réjouissant d'entendre un monteur critiquer mon travail, et ce à l'intérieur d'un de mes propres films.

JPG : Il t'engueule!

PC : Il critique mon travail, en effet! Mais attention, ça pourrait être une critique anecdotique, pour de faux, complaisante quoi. On n'évite pas toujours la complaisance quand on met en scène notre propre travail. On a pris ce risque. Je ne suis pas sûr que ça soit très concluant mais j'avais envie de le tenter. Puis j'avais envie de filmer ce monteur, Roger Ikhlef, qui a été quelqu'un d'important pour moi. Je voulais lui rendre hommage. Et il me semblait qu'il avait sa place dans ce film où il était question des derniers des Mohicans, où l'on filmait des activités en voie de disparition dans la société actuelle ou, du moins, la manière dont on les exerce. Bref, Roger Ikhlef n'apparaissait pas comme un extra-terrestre dans ce film.

\section{Le montage, c'est toujours un compromis entre deux tentations : guider ou laisser libre}

En tant que réalisateur, on est tenté de manipuler le spectateur, de le conduire dans une direction précise, de l'amener à rire à certains moments, à s'émouvoir à d'autre. Dans le même temps, le réalisateur que je suis est aussi un spectateur de films et, en tant que spectateur, il n'aime pas être manipulé. On est partagé : on aimerait laisser le spectateur être le plus libre possible, parce qu'en tant que spectateur on aime qu'un film ne nous dise pas ce qu'il faut penser, ne nous prenne pas trop par la main. On préfère déambuler, picorer, se balader à notre guise et à notre rythme dans ce tissu d'images et de sons. J'éprouve beaucoup plus de plaisir en tant que spectateur lorsque je me sens actif. Et en même temps, le réalisateur qui est en moi a envie de mettre le spectateur sur des rails, de le conduire d'un point $\mathrm{A}$ du récit à un point $\mathrm{B}$ en passant par un point $C$, de le guider, de le manipuler. Pour le réalisateur, il y a une tentation d'abus de pouvoir alors qu'en tant que spectateur, il y a une envie de ne pas être victime de cet abus de pouvoir. On est confronté à ce dilemme.

Qui l'emporte ? Ça dépend des films. Dans ma filmographie, il y a des documentaires comme Pas vu, pas pris qui guident énormément le spectateur. Tu as très peu de liberté, ce qui ne t'empêche pas d'éprouver un certain plaisir en voyant le film et en lançant des tomates à la tête de ces journalistes vedettes. Et puis, il y a d'autres films, comme Volem rien foutre al païs ou La Sociologie est un sport de combat, où le spectateur est laissé relativement libre d'interpréter ce qu'il a sous les yeux. C'est plus déroutant pour lui au premier abord mais probablement plus intéressant au final. Il a le sentiment, ce n'est pas qu'un sentiment, d'être actif.

On doit à la fois proposer un spectacle, car c'est la raison pour lesquelles les gens vont au cinéma : pour s'en prendre plein les yeux, assister à un spectacle forain comme 
cela a été le cas depuis les origines du cinéma, et essayer de faire en sorte que le spectateur ne soit pas trop infantilisé. Il faut qu'il puisse se poser un certain nombre de questions, qu'il soit en mesure même d'exercer son esprit critique. Il faut essayer de lui laisser cette place-là. Au montage, tu es toujours tiraillé entre : « emmenons-le là » et "c'est dégueulasse de l'amener là ». Voilà, le conflit qui nous traverse. Et chaque fois, tu te demandes si tu vas réussir à ne pas abuser de ton pouvoir, en tant que réalisateur.

\section{Je fais un cinéma sans contraintes extérieures sauf celles que j'intègre moi même sans en avoir conscience}

Mes films sont produits la plupart du temps par Annie Gonzalez et C-P Productions, une petite société créé par des travailleurs de l'audiovisuel il y a une quinzaine d'années. Elle n'a pas intégré les contraintes de la télévision étant donné que cette dernière ne finance pas notre travail. Nous ne sommes pas obligés de faire un 26 minutes, un $52 \mathrm{mn}$, un $90 \mathrm{~min}$, avec une voix off ou de la musique omniprésente. Je n'utilise pas de musique dans mes films, exception faite du générique de fin, alors que l'on sait à quel point la musique est utilisée la plupart du temps pour susciter des émotions, pour surligner à la manière d'un stabylo boss au cas où le spectateur n'aurait pas compris ce qui se passe, ce qui est là encore une manière de l'infantiliser une fois de plus. Ainsi, le choix de guider plus ou moins le spectateur ne dépend que de nous, des gens avec qui je travaille. Nous ne pouvons pas nous réfugier derrière des donneurs d'ordre, des commanditaires, des responsables de documentaires des chaînes de télévision. On ne peut pas dire que c'est eux qui nous ont imposé ceci ou cela. Nous sommes seuls responsables. Ou presque.

Mais le fait de ne pas être produit par la télévision ne suffit pas pour faire quelque chose d'intéressant en matière de documentaire. Dans le domaine de la fiction, par exemple, il y a des films qui ont obtenu l'avance sur recettes su CNC (Centre National du cinéma et de l'image animée) et qui se conforme au bon goût auteuriste ou aux canons des festivals. Tu sens que le réalisateur ou le producteur ont fabriqué leur film pour qu'il soit bien vu par un certain public, celui des festivaliers, des jurys de festivals, des commissions du CNC... Il y a tout un cinéma de festival qui se présente comme libre alors qu'il est corseté et assez normé, répondant à des règles, notamment esthétiques très précises, à des contraintes non dites, alors même qu'il prétend s'en affranchir.

JPG : Tu insistais sur le côté narration, sur le côté passeur, sur la nécessité de raconter une histoire. Tu es aussi et peut être surtout un conteur. Comment à partir de cette vérité relative qu'est l'entretien on passe à un film, à un spectacle... forain tu disais.

PC : Je parlais de la dimension foraine, parce qu'il est passé un contrat tacite avec le spectateur : celui de lui faire éprouver des émotions qui ne sont pas si éloignées de celle des origines du cinéma. "Vous allez en prendre plein les yeux». On peut difficilement ne pas proposer ce parcours au spectateur parce qu'il est inscrit dans l'inconscient collectif, me semble-t-il. Quand on va voir des images, on s'attend quelque part à cela. Après on peut frustrer le spectateur, bien sûr. 


\section{C'est très excitant de se dire qu'avec des images on peut modifier le réel}

Quand on fait des films qui donnent la parole à des gens qu'on entend peu ou pas, on se dit que ça va faire son chemin chez le spectateur et qu'il sera peut-être amené à s'emparer de ces propos, de ces pensées et, au final, à vouloir changer le monde, le sien pour commencer. Oui, il y a une volonté d'action politique qui transparait dans mon travail. Je suis d'ailleurs pas sûr d'être véritablement un réalisateur. Plutôt un agitateur politique...

JPG : Tu es un agitateur?

PC : Oui, je fais de l'agit-prop d'une certaine manière. Et je crois au côté performatif des discours : tu racontes des choses pour qu'elles se produisent. Tu mets dans la tête des spectateurs des idées, des propos, des images qui peuvent être de l'utopie, de l'anticipation, de la prospective, mais avec l'idée que cela deviendra un jour réalité. C'est évident avec le tout dernier film Opération Correa : cette fois-ci, ce que raconte Correa n'est pas une fiction, ça a déjà un début de réalité en Equateur. Mais on a envie que ça fasse tache d'huile, que ça contamine, que ça ne reste pas simplement cantonné à l'Amérique latine. Il y a clairement une intention d'action politique dans ce genre de film. Le prochain épisode est en cours de fabrication. On essayera d'aller plus loin dans ce domaine, d'interférer vraiment sur la réalité politique française avec nos prochains films. Nous avons l'avantage de ne pas être soumis aux règles du CSA. Nous pouvons sortir des films quelques mois avant l'élection présidentielle, sans devoir respecter les règles d'égalité auxquelles sont tenues les chaînes de télévision. Sur le petit écran, il faut donner le même temps de parole aux uns et aux autres, du moins en fin de la campagne car le reste du temps les grands médias audiovisuels donnent surtout la parole aux puissants ou disons aux candidats qui ne menacent pas les intérêts du pouvoir économique. Nous n'y sommes même pas obligés.

\section{Le cinéma documentaire ne doit pas se limiter au constat, même si il m'est arrivé de faire des films de ce type}

Je pense au travail que j'ai fait lorsque je travaillais pour l'émission franco-belge Strip-Tease. Le petit film Pizza americana est un film-constat: voilà comment se passent les choses dans un secteur professionnel lorsqu'on introduit la culture d'entreprise étasunienne. Même si ce travail audiovisuel porte un regard critique, je me demande jusqu'à quel point il n'est pas finalement conformiste. Lorsqu'il n'y a pas de dimension utopique, lorsque les films se limitent à montrer des mécanismes d'exploitation, d'aliénation, je me demande s'ils ne contribuent pas finalement à reproduire l'ordre établi. Notre société du spectacle est capable de faire du spectacle y compris avec cela à condition qu'on n'imagine pas d'alternative au système capitaliste d'exploitation. II y a aujourd'hui tout un genre dans le documentaire qui déplore ce qui se passe dans cette société, qui déplore la souffrance sociale, qui déplore les relations de domination, mais qui peut devenir un produit de consommation. Et qui ne dérange pas tant que cela le système au final.

Il y a un genre à part entière qui a vu le jour à la télévision, ce sont les documentaires ou les téléfilms sur le combat de travailleurs pour éviter la fermeture de leur usine et la sauvegarde de leur emploi. Le discours peut aller jusqu'à « Regardez comment les méchants patrons ce comportent mal avec leurs salariés ». Tout un tas de films vont 
dans ce sens-là. Mais tant que le discours implicite reste de l'ordre de « les politiques néo-libérales créent ce type de dommage mais n'imaginez pas d'autres politiques possibles" tout cela reste assez inoffensif. Ce sont ces films que j'appelle " constatatifs ", je ne sais pas si le terme existe. Ils se contentent d'établir un constat. S'ils s'en tiennent à ce constat, ils peuvent être très conservateurs finalement, à la fois déprimants et incitant à une certaine forme de résignation.

J'essaie au contraire dans mes films de montrer qu'il peut en être autrement, qu'il y en est déjà autrement ailleurs, en Equateur par exemple. Si ça peut se faire ailleurs ou si ça a pu se faire à d'autres périodes de l'histoire, il n'y a pas de raison que ça ne se reproduise pas. Il ne s'agit pas de simples incantations.

JPG : Pour toi, le cinéma peut changer le monde?

\section{Il est vital de produire des films coûte que coûte, de les fabriquer même si le système n'en veut pas et ne donne pas les moyens pour qu'ils existent}

PC : Plus que le cinéma, c'est l'utopie dans laquelle les gens peuvent se projeter. Si tu peux te projeter dans un récit à caractère utopique en sachant que, dans l'histoire de l'humanité, il y a eu ces moments où tout semblait verrouillé, bloqué, où l'on pensait que rien ne pouvait changer jusqu'à ce qu'advienne une révolution... Si il y avait des films, si il y avait des fictions, si il y avait des images, si il y avait des projections qui nous permettaient d'envisager d'autres sociétés que celle dans laquelle nous évoluons actuellement, peut-être que l'on aurait envie de jouer un rôle dans un tel film.

JPG : C'est un aspect qu'on n'a pas abordé, qui est quand même important à mon avis dans ton cinéma, c'est l'utopie. Et faire parler les penseurs d'utopie.

PC : Prenons l'exemple de la lutte armée en Colombie. A l'heure où je parle, il y a encore 10000 personnes qui sont dans la jungle colombienne à ne pas avoir encore déposé les armes, à réclamer une réforme agraire, à se battre pour la fin des injustices sociales en Colombie. Même si ça peut paraître des situations à des années lumières de nous, c'est quand même réjouissant de savoir qu'un maquis traversé d'idéaux communistes subsiste sur la planète. On pourrait aussi parler du PKK (Parti des Travailleurs du Kurdistan) qui est aussi un groupe armé luttant pour une société plus égalitaire. Or la seule représentation des groupes armés luttant contre des Etats que nous avons à travers les grands médias, c'est Al Qaida, c'est Daesh... On met dans le même sac tous les dits terroristes. Non, il y a d'autres types de luttes armées que celle de Daesh, où il n'est pas question de religion mais de lutte des classes. C'est bien de le rappeler.

JPG : Un dernier point peut-être. Ton cinéma est relativement collectif. Même si bon, tu es devant. Est-ce que Pierre Carles fait école. Moi je le crois, c'est pour ça que je te pose la question.

PC : Je ne sais pas. Ce serait de la fausse modestie de dire que je ne m'en préoccupe pas et que je ne suis pas attentif à cela. Je crois que ma démarche peut faire école. Pas tant sur le contenu que sur la fait de produire des films coûte que coûte, de fabriquer ce type d'objets audiovisuels même si le système n'en veut pas. Savoir que l'on peut toujours se débrouiller, bidouiller, bricoler, passer par la fenêtre quand on s'est fait virer par la porte. Je ne suis pas le seul, il y a d'autres réalisateurs qui s'inscrivent 
dans cette démarche et dont les travaux sont bien antérieurs aux miens. Je pense notamment à Luc Moullet ou à Pierre Merejkowsky...

Avec C-P Productions, on a prouvé qu'il était possible, avec peu de moyens, en se regroupant pour monter un collectif de production documentaire, en s'appuyant sur les quelques salles de cinéma indépendantes et aussi les internautes, sans rien attendre de la télévision mais sans cracher non plus sur les financements institutionnels, de fabriquer tous ces films. Ceux-ci ont rencontré un certain succès public, une certaine audience; ils circulent. C'est possible, alors que ça pouvait paraître impossible à une époque où il fallait être dans le système et passer sous les fourches caudines de la télévision. Nos films prouvent qu'il y a possibilité de faire des films qui rencontrent leur public avec peu de moyens. Mais il ne faut pas généraliser non plus. Ça reste de l'ordre de l'exception, ce n'est pas facile à reproduire. L'expérience de C-P production, le travail d'Annie Gonzalez, le mien, celui des gens avec qui je travaille, comme Nina Faure ou d'autres montrent qu'il y a une lueur d'espoir. Des jeunes viennent nous proposer leurs services parce qu'ils voient que nous comportons aussi comme de sales garnements : ce n'est pas parce que certaines choses sont interdites, paraissent impossibles à faire, qu'on ne va pas... les faire !

\section{AUTEURS}

\section{JEAN-PAUL GÉHIN}

Jean-Paul Géhin est enseignant chercheur à l'Université de Poitiers et membre du GRESCO. Il a publié de nombreux articles et ouvrages en sociologie du travail et plus particulièrement sur la question des modalités d'articulation entre éducation et travail. Responsable et enseignant dans des masters de réalisation documentaire, il s'intéresse à la médiation scientifique et à l'usage des images en sciences sociales. Dans ce cadre, il a participé à la fondation en 2009 du festival Filmer le travail qu'il préside actuellement. Il fait également partie de la direction collégiale de la nouvelle revue numérique et pluridisciplinaire Images du travail, travail des images.

\section{MAXENCE LAMOUREUX}

(cadre et montage)

Maxence Lamoureux est docteur de l'Université de Poitiers, sa thèse consacrée à la sociologie du monde des cinéastes animaliers français, a été soutenue en septembre 2015. Il est par ailleurs, réalisateur de documentaires sociaux et nature.

Communications :

" L'indépendance en question dans le groupe professionnel des cinéastes animaliers", Congrés de l'AFS 2013, RT1

"Jacques Perrin ou l'exigence qualitative et populaire. La démarche originale du réalisateur et producteur de films animaliers pour le cinéma", Colloque organisé par "Jacques Perrin, portrait d'un artiste engagé", Avril 2014, RIRRA 21, l’Université de Montpellier 3. 